Rev. Bras. Saúde Prod. Anim., Salvador, v.17, n.2, p.195-201 abr./jun., 2016 http://www.rbspa.ufba.br

\title{
Processos térmicos em dietas para frangos de corte na fase pré-inicial
}

\author{
Thermal processes in diets for broiler in the pre-starter phase
}

\author{
BOEMO, Lenise Schroder ${ }^{1 *}$; ROSA, Daniele Pozzebon da $^{1}$; ROSA, Alexandre Pires ${ }^{1}$; \\ ORSO, Catiane ${ }^{1}$; SCHER, Anelcir ${ }^{1}$; GEHRKE, Sandra Bonaspetti²; SILVA, Adrian \\ Ertman $^{1}$; MARIANI, Alexandre Bonadiman ${ }^{1}$
}

\author{
${ }^{1}$ Universidade Federal de Santa Maria, Centro de Ciências Rurais, Departamento de Zootecnia, Santa \\ Maria, Rio Grande do Sul, Brasil. \\ ${ }^{2}$ JBS FOODS, Itajaí, Santa Catarina, Brasil. \\ *Endereço para correspondência: lensieboemo@hotmail.com
}

\section{RESUMO}

O objetivo neste estudo foi avaliar o desempenho zootécnico de frangos de corte de 1 a 7 dias de idade alimentados com a inclusão de $20 \%$ farelo de arroz parboilizado (FAP) na dieta com diferentes processamentos. O delineamento utilizado foi em esquema fatorial $2 \times 3$, dois tipos de dietas (uma a base de milho e a outra com a inclusão de $20 \%$ FAP) e três tipos de processamento: dietas farelada, peletizada e expandida peletizadas todas isonutritivas. As rações foram peletizadas a uma temperatura de $85^{\circ} \mathrm{C}$ e expandidas em uma faixa de temperatura de $110^{\circ} \mathrm{C}$ a $115^{\circ} \mathrm{C}$. O estudo foi realizado em aviário experimental composto por 60 boxes experimentais. Cada unidade experimental foi composta por 58 pintos de corte machos da linhagem Cobb $500 \AA$ de um dia de idade. Foram utilizados seis tratamentos com 10 repetições por tratamentos. Foram avaliadas as variáveis de desempenho aos 7 dias de idade. As médias quando apresentaram diferenças a nível de $5 \%$, foram comparadas pelo teste de Tukey. As variáveis analisadas não apresentaram interação significativa $(\mathrm{P}<0,05)$ no período avaliado. Pintos de corte alimentados com a inclusão de $20 \%$ FAP na dieta apresentaram menor peso corporal, ganho de peso e consumo de dieta aos 7 dias de idade. O processo de expansão + peletização promove melhora no peso aos 7 dias de idade e no ganho de peso dos pintos até os 7 dias de idade.

Palavras-chave: desempenho de frangos de corte, expansão, farelo de arroz parboilizado, peletização

\section{SUMMARY}

The aim of this study was to evaluate the growth performance of broiler chickens 1-7 days old fed the inclusion of $20 \%$ parboiled rice bran (PRB) in the diet and different processes. The design utilized was in a $2 \times 3$ factorial, two types of diets (one based on corn and the other with the inclusion of $20 \%$ PRB) and three types of processing, with mash diets, pelleted and expanded pelleted, all isonutritives. Diets were pelleted at a temperature of $85^{\circ} \mathrm{C}$ and expanded in a temperature range of $110^{\circ} \mathrm{C}$ to $115^{\circ} \mathrm{C}$. The study was conducted in experimental avian composed of 60 boxes experimental. Each experimental unit consisted of 58 male broiler chicks of the Cobb 500 ( ) line of one day old. Six treatments with 10 repetitions per treatment were used. The performance variables at 7 days of age were evaluated. Average when presented differences in 5\% were compared by Tukey test. The variables analyzed showed no significant interaction $(\mathrm{P}<0.05)$ during the study period. Broiler chicks fed the inclusion of $20 \%$ PRB diet had lower body weight, weight gain and diet consumption at 7 days old. The expansion+ pelleted process promotes improvement in weight at 7 days of age and weight gain of the chickens until 7 days age.

Keywords: performance of broiler chickens, expansion, parboiled rice bran, pelletizing 


\section{INTRODUÇÃO}

O período pós-eclosão é crítico para o crescimento das aves. As limitações fisiológicas para o aproveitamento de nutrientes nos primeiros dias de vida dos pintos e a importância desse período para o sucesso no desempenho dos frangos de corte têm levado a estudos sobre o uso de dietas especiais para a primeira semana de vida (FREITAS et al., 2005). Nessa fase se torna de fundamental importância o fornecimento de alimentos de alta qualidade e disponibilidade de nutrientes.

$\mathrm{O}$ processamento térmico de dietas visa melhorar as características físicas do alimento bem como seu valor nutricional. O processo de peletização é utilizado na indústria avícola e seus benefícios são consolidados, principalmente no seu acréscimo do valor nutricional pela possível melhora na digestibilidade de nutrientes (GADZIRAYI et al., 2006; FREITAS et al., 2008).

Além da peletização, a expansão é outro processamento que vem crescendo na produção de dietas avícolas. Este processo é usado principalmente como pré-tratamento de dietas antes da peletização. A expansão em comparação com o processo de peletização tem como objetivos aumentar a gelatinização do amido facilitando a digestibilidade, melhorar a durabilidade do pellet, destruir fatores anti-nutricionais, destruir agentes patogênicos, e melhorar a incorporação de gordura adicionada (LÓPEZ et al., 2007).

Além da forma física e tipo de processamento empregado em dietas pré iniciais, outro fator de grande importância é o tipo de ingrediente utilizado. O farelo de arroz, subproduto do polimento ou beneficiamento do arroz, mostra-se como ingrediente alternativo para alimentação de aves. O farelo de arroz parboilizado (FAP), obtido após o processo de parboilização não necessita de tratamento térmico adicional para ser utilizado na alimentação, uma vez que este processo pode inativar enzimas responsáveis pela degradação dos lipídios (SILVA et al., 2006).

$\mathrm{O}$ objetivo neste estudo foi avaliar o desempenho zootécnico de frangos de corte de 1 a 7 dias de idade alimentados com a inclusão de $20 \%$ farelo de arroz parboilizado (FAP) na dieta com diferentes processamentos.

\section{MATERIAIS E MÉTODOS}

Este experimento foi realizado no Laboratório de Avicultura da Universidade Federal de Santa Maria (UFSM) e seguiu as diretrizes da Comissão de Ética no uso de Animais da UFSM, sob protocolo número 7800310315. Foram utilizados 3480 pintos de corte machos de um dia de idade da linhagem Cobb 500®, com peso médio de 44g. O período experimental compreendeu a fase pré-inicial (1 a 7 dias de idade).

Foi utilizado delineamento experimental inteiramente casualizado em esquema fatorial $(2 \times 3)$, dois tipos de dietas $(20 \%$ de FAP e dieta convencional) e três tipos de processamento (farelada, peletizada, expandida peletizada) totalizando seis tratamentos com 10 repetições, sendo a unidade experimental composta por 58 aves. As aves foram mantidas dentro da zona de termoneutralidade recomendada pelo manual de criação da linhagem, onde o aquecimento foi realizado por meio de campanulas elétricas. $\mathrm{O}$ programa de iluminação utilizado foi constante com 24 horas de luz.

As dietas experimentais foram formuladas para atender as exigências nutricionais, de acordo com a recomendação de Rostagno et al. (2005). A composição dos seis tratamentos consistiu: Dieta sem tratamento térmico na forma física farelada a base de milho e farelo de soja (dieta convencional), dieta convencional peletizada a $85^{\circ} \mathrm{C}$, dieta convencional expandida peletizada na faixa de temperatura de 110 a $115^{\circ} \mathrm{C}$, dieta sem tratamento térmico na forma física farelada com a inclusão de $20 \%$ de FAP, dieta 
peletizada a $85^{\circ} \mathrm{C}$ com a inclusão de $20 \%$ de FAP, dieta expandida peletizada na faixa de temperatura de 110 a $115^{\circ} \mathrm{C}$ com a inclusão de $20 \%$ de FAP. O FAP possuindo composição bromatológica de $89,00 \%$ de matéria seca, $13,50 \%$ de proteína bruta, 7,90 de fibra bruta e $3.050 \mathrm{kcal} / \mathrm{kg}-1$ de energia metabolizável.

As dietas foram processadas em uma única linha de produção de uma fábrica de rações, sendo esta equipada com expander e peletizadora. As dietas foram isonutritivas, ocorrendo apenas variações entre os tipos de ingredientes $\mathrm{e}$ de processamento.

Tanto as dietas peletizadas como as expandidas peletizadas, foram submetidas a uma temperatura de $85^{\circ} \mathrm{C}$ no condicionador. As dietas expandidas peletizadas foram expandidas em uma faixa de temperatura de 110 a $115^{\circ} \mathrm{C}$, com a inclusão de $1,5 \%$ de água no condicionador. $\mathrm{O}$ tempo que a mistura permaneceu no condicionador e no retentor foi de 50 segundos.

Após a peletização, a dieta foi resfriada a uma temperatura inferior ou igual a $22^{\circ} \mathrm{C}$. Dietas que passaram pelo processo de peletização foram trituradas, com a finalidade de diminuir o tamanho do pellet e facilitar a apreensão do alimento pelas aves. A composição nutricional e centesimal das dietas se encontram na Tabela1.

Tabela 1. Composições centesimais e químicas das dietas fornecidas às aves no período de 1 a 7 dias

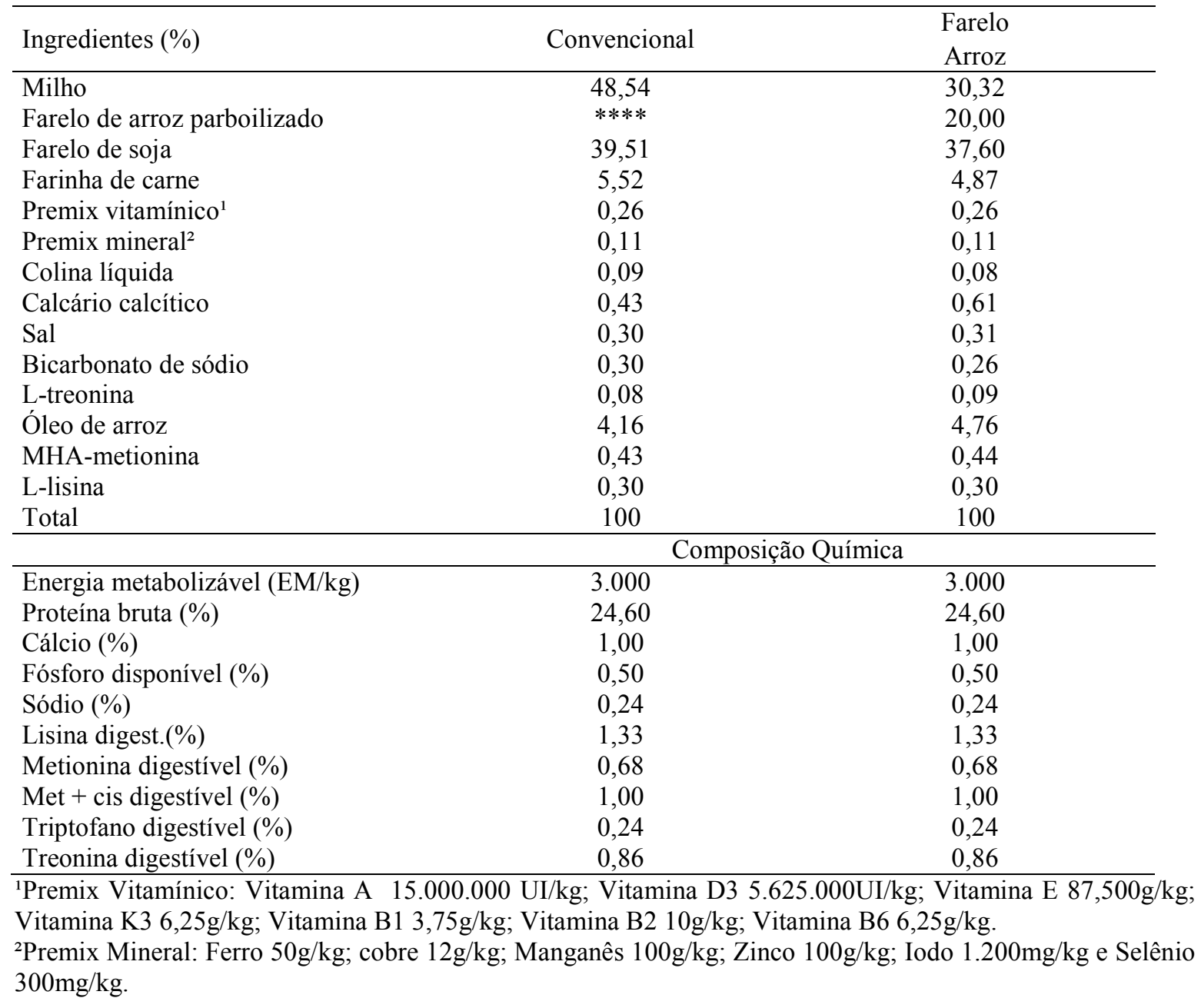


Rev. Bras. Saúde Prod. Anim., Salvador, v.17, n.2, p.195-201 abr./jun., 2016 http://www.rbspa.ufba.br ISSN 15199940 http://dx.doi.org/10.1590/S1519-99402016000200007

As variáveis analisadas de desempenho zootécnico foram: peso corporal (PC), ganho de peso corporal (GP), consumo de dieta $(\mathrm{CO})$ e conversão alimentar (CA) mensuradas ao final da fase. A mortalidade foi registrada diariamente $\mathrm{e}$ analisada ao final do período. Os dados foram submetidos à análise de variância e teste F. Quando ocorreram diferenças significativas ao nível de $5 \%$ de significância, foi aplicado o teste de comparação de médias de Tukey. As análises foram realizadas com $\mathrm{o}$ programa SAS (2001).

\section{RESULTADOS E DISCUSSÃO}

Não houve interação significativa entre os fatores estudados no período avaliado, porém algumas variáveis apresentaram diferenças significativas (Tabela 2).

Tabela 2. Variáveis de desempenho de pintos de corte machos alimentados com dietas contendo $20 \%$ de FAP e submetidas a diferentes processamentos*

\begin{tabular}{|c|c|c|c|c|c|}
\hline \multirow{3}{*}{ Fator } & \multicolumn{5}{|c|}{ Variáveis de Desempenho } \\
\hline & Peso (g) & $\begin{array}{l}\text { Ganho de } \\
\text { peso }(\mathrm{g})\end{array}$ & $\begin{array}{l}\text { Consumo } \\
\text { ração }(\mathrm{g})\end{array}$ & $\begin{array}{l}\text { Conversão } \\
\text { alimentar }\end{array}$ & $\begin{array}{c}\text { Mortalidade } \\
(\%)\end{array}$ \\
\hline & 7 dias & $1-7$ dias & $1-7$ dias & 1-7 dias & $1-7$ dias \\
\hline \multicolumn{6}{|l|}{ Dieta } \\
\hline Convencional & $194,97^{\mathrm{a}}$ & $150,33^{\mathrm{a}}$ & $155,97^{\mathrm{a}}$ & 1,04 & 0,80 \\
\hline $20 \%$ FAP & $191,37^{\mathrm{b}}$ & $146,95^{\mathrm{b}}$ & $151,70^{\mathrm{b}}$ & 1,03 & 0,74 \\
\hline \multicolumn{6}{|l|}{ Processo } \\
\hline Farelada & $187,90^{\mathrm{c}}$ & $143,36^{\mathrm{c}}$ & $152,00^{b}$ & $1,06^{\mathrm{a}}$ & 1,12 \\
\hline Peletizada & $193,75^{\mathrm{b}}$ & $149,19^{b}$ & $156,21^{\mathrm{a}}$ & $1,05^{\mathrm{a}}$ & 0,73 \\
\hline Expandida & $198,86^{\mathrm{a}}$ & $154,36^{\mathrm{a}}$ & $153,59^{b}$ & $0,99^{\mathrm{b}}$ & 0,41 \\
\hline \multicolumn{6}{|l|}{ Dieta * Processo } \\
\hline Convencional Frelada & 190,53 & 145,97 & 154,92 & 1,06 & 1,38 \\
\hline Convencional Peletizada & 194,66 & 149,98 & 156,71 & 1,05 & 0,52 \\
\hline Convencional Expandida & 200,89 & 156,21 & 156,34 & 1,00 & 0,43 \\
\hline $20 \%$ FAP Farelada & 185,27 & 140,75 & 149,08 & 1,06 & 0,86 \\
\hline 20\% FAP Peletizada & 192,61 & 148,21 & 155,58 & 1,05 & 0,99 \\
\hline 20\% FAP Expandida & 197,06 & 152,72 & 151,15 & 0,99 & 0,38 \\
\hline \multicolumn{6}{|l|}{ Fonte de Variação } \\
\hline Dieta & $<, 0001$ & $<, 0001$ & $<, 0001$ & 0,6446 & 0,9536 \\
\hline Processo & $<, 0001$ & $<, 0001$ & $<, 0001$ & $<, 0001$ & 0,1769 \\
\hline Dieta * Processo & 0,174 & 0,1475 & 0,0133 & 0,5542 & 0,5796 \\
\hline Medias & 193,50 & 148,97 & 153,96 & 1,03 & 0,76 \\
\hline $\mathrm{CV} \%$ & 1,29 & 1,61 & 1,59 & 2,08 & 1,39 \\
\hline
\end{tabular}

*Médias seguidas por letras distintas na mesma coluna diferem entre si pelo teste de Tukey $(\mathrm{P}<0,05)$.

No estudo do fator dieta as aves alimentadas com dieta convencional apresentaram maior PC e GP em relação as aves que receberam dieta contendo $20 \%$ FAP. O farelo de arroz possui em sua composição fibras que são ricas em polissacarídeos não amiláceos. Estes diminuem a taxa de passagem dos alimentos e reduzem a digestão e absorção de lipídios, uma vez que em ambiente viscoso, o contato entre as gorduras e secreções digestivas, é menor 
(VIEIRA et al., 2007; KRAS et al., 2013). O exposto pode explicar o menor PC e GP das aves alimentas com $20 \%$ FAP.

Aves alimentadas com dietas expandidas peletizadas, apresentaram maior $\mathrm{PC} \mathrm{e}$ GP do que as que receberam dietas apenas peletizadas. Já as aves alimentadas com dietas na forma farelada apresentaram os menores resultados de $\mathrm{PC}$ e GP no período avaliado. $\mathrm{O}$ maior $\mathrm{PC}$ das aves que receberam dietas na forma expandida peletizada, está ligado às transformações químicas que o processo realiza no alimento, como o aumento na gelatinização do amido, proporcionando melhor aproveitamento dos nutrientes pelo animal. Resultado semelhante foi encontrado por Oliveira et al. (2011) que constataram que frangos de corte alimentados com dietas expandidas peletizadas apresentam maior GP no período de 1 a 21 dias do que as aves alimentadas com dietas fareladas.

O CO das aves alimentadas com $20 \%$ de FAP foi inferior ao das aves que receberam dieta convencional. De acordo com Souza \& Lopes (1994), o fato de maiores níveis de inclusão de farelo de arroz determinar prejuízos ao desempenho, está associado à diminuição no consumo, o que ocorre em função da baixa digestibilidade dos polissacarídeos não-amiláceos. Bedford et al. (1991) também verificaram redução no consumo de dieta de aves alimentadas com dietas contendo $20 \%$ de FAP.

Aves alimentadas com dietas peletizadas apresentaram $\mathrm{CO}$ superior àquelas que foram alimentadas com dietas fareladas ou expandidas peletizadas. Esse resultado não corrobora com estudos realizados por Oliveira et al. (2011), que avaliaram diferentes processamentos em dietas para frangos de corte, mostraram que as aves alimentadas com dietas expandidas peletizadas apresentaram maior consumo de ração no período inicial em comparação a aves alimentadas com dietas peletizadas.

$\mathrm{O}$ fator processamento apresentou diferenças significativas na $\mathrm{CA}$ dos pintos no período avaliado $(\mathrm{P}<0,0001)$, onde as aves que consumiram dieta na forma expandida apresentaram melhor CA que as aves que receberam dietas na forma farelada ou peletizada. $\mathrm{O}$ tratamento térmico de dietas aumenta a eficiência de retenção da energia metabolizável aparente, sendo essa melhor eficiência justificada pela melhora na $\mathrm{CA}$ em frangos de corte (LEMME et al., 2006). No estudo de González-Alavarado et al. (2007), o tratamento térmico do cereal melhorou a utilização de nutrientes em dietas convencionais na fase inicial de frangos de corte.

A mortalidade não apresentou diferenças significativas entre os fatores avaliados. Esse fato pode ser atribuído ao período avaliado (pré-inicial), onde ainda não se evidenciam casos de mortalidade por ascite ou síndrome da morte súbita que são atribuídos ao aumento da ingestão de nutrientes na dieta ocorridos pelo tratamento térmico (CAPDEVILA, 1997), e por as aves ainda estarem protegidas pela imunidade materna.

Conclui-se que a inclusão de $20 \%$ de FAP na dieta afetou negativamente $o$ peso corporal das aves no período avaliado. Já o uso do processo de expansão em conjunto com a peletização promove melhora nos índices de desempenho de pintos de corte na fase pré-inicial de produção (1 a 7 dias).

\section{AGRADECIMENTOS}

Á empresa JBS Foods pela doação dos pintos e dietas para a realização deste estudo. 
Rev. Bras. Saúde Prod. Anim., Salvador, v.17, n.2, p.195-201 abr./jun., 2016 http://www.rbspa.ufba.br ISSN 15199940

\section{REFERÊNCIAS}

BEDFORD, M.R.; CLASSEN, H.L.; CAMPBELL, G.L. The effect of pelleting, salt and pentosanase on the viscosity of intestinal contents and the performance of broilers fed ryebased diets. Poultry Science, v.70, n.7, p.15717, 1991.

CAPDEVILA, J. Efectos de la granulación sobre la formulación de raciones em avicultura. Selecciones Avícola, v.39, p.465-474, 1997.

FREITAS, E.R.; SAKOMURA, N.K.; NEMES, R.; BOARBOSA N.A.A. Valor nutricional do milho termicamente processado usado na ração pré-inicial para frangos de corte. Arquivos Brasileiros de Medicina Veterinária e Zootecnia, v.57, n.4, p.510-517, 2005.

FREITAS, E.R.; SAKOMURA, N.K.; DAHLKE, F.; SANTOS, F.R.; BOARBOSA N.A.A. Desempenho, eficiência de utilização dos nutrientes e estrutura do trato digestório de pintos de corte alimentados na fase pré-inicial com rações de diferentes formas físicas.

Revista Brasileira de Zootecnia, v.37, n.1, p.73-78, 2008.

GADZIRAYI, C.T.; MUTANDWA, E.; CHIHIYA, J.; MLAMBO, R. A Comparative Economic Analysis of Mash and Pelleted Feed in Broiler Production under Deep Litter Housing System. International Journal of Poultry Science, v.7, p.629-631, 2006.

GONZÁLEZ-ALVARADO, J.M.; JIMÉNEZ-MORENO, E.; LÁZARO, R.; MATEOS, GG. Effect of type of cereal, heat processing of the cereal, and inclusion of fiber in the diet on productive performance and digestive traits of broilers. Poultry Science, v.86, p.17051715, 2007.
KRAS, R.V.; KESSLER, A. de M.; RIBEIRO, A.M.L.; HENN, J.D.; BOCKOR, L.; SBRISSIA, A.F. Effect of dietary fiber, genetic strain and age on the digestive metabolism of broiler chickens. Revista Brasileira de Ciencia Avicola, v.15, n.2, p.83- 90, 2013.

LEMME, A.; WIJTTEN, P.J.A.; VAN WICHEN, J. Responses of male growing broilers to increasing levels of balanced protein offered as coarse or pellets of varying quality. Poultry Science, v.85, p.721-730, 2006.

LÓPEZ, C.A.A; BAIÃO, N.C.; LARA, L.J.C.; RODRIGUEZ, N.M.; CANÇADO, S.V. Efeitos da forma física da ração sobre a digestibilidade dos nutrientes e desempenho de frangos de corte. Arquivos Brasileiros de Medicina Veterinária e Zootecnia, v.59, n.4, p.1006-1013, 2007.

OLIVEIRA, A.A. de; GOMES, A.V.da C.; OLIVEIRA,G.R.de.; LIMA, M.F.DE., DIAS,G.E.A.; AGOSTINHO, T.S.P.; SOUSA, F.D. de R.; LIMA,C.A.R. de. Desempenho e características da carcaça de frangos de corte alimentados. Revista Brasileira de Zootecnia, v.40, n.11, p.2450-2455, 2011 .

ROSTAGNO, H.S. Tabelas brasileiras para aves e suínos: composição de alimentos e exigências nutricionais. Viçosa, MG: UFV, 2005. 141p.

SILVA, M.A.; SANCHES, C.; AMANTE, E.R. Prevention of hydrolytic rancidity in rice bran. Journal of Food Engineering, v.75, n.4, p.487-491, 2006. 
SOUZA, G.A.; LOPEZ, J. Farelo de arroz integral como fonte de fósforo em rações para frangos de corte.1.

Desempenho e produtividade animal. Revista da Sociedade Brasileira de Zootecnia, v.23, n.1, p.73-84, 1994.

SAS. Statistical Analysis System. System for Microsoft Windows: Release 8.2. Cary, 2001.

VIEIRA, A.R.; RABELLO, C.B.V.; LUDKE, M. do C.M.M.; DUTRA JUNIOR, W.M.; TORRES, D.M.;

LOPES J.B. Efeito de diferentes níveis de inclusão de farelo de arroz em dietas suplementadas com fitase para frangos de corte. Acta Scientiarum. Animal Sciences, v.29, n.3, p.267-275, 2007.

Data de recebimento: 08/09/2015

Data de aprovação: 02/05/2016 